\title{
TURNING UP THE HEAT - USER ERRORS AND OTHER INSIGHTS INTO OPTION SELECTION
}

\author{
Mairéad Hogan and Chris Barry \\ NUI Galway. Galway, Ireland
}

\begin{abstract}
This paper presents a research project that used eye tracking technology to study optionality within the online transactional process. The focus was on how micro-decisions can be framed; the option type; the impact of decision default values; and the types of decision constructs. It elaborates and improves on a pilot study that was used to test the experiment design. Prior research that identified problematic decision constructs informed the types of decisions studied. The main findings relate to participant task error rates. Heat maps have also been used to further illustrate the findings.
\end{abstract}

\section{KEYWORDS}

Micro-Decision, Opt-In, Opt-Out, Framing, Default Effect, Eye Tracking

\section{INTRODUCTION}

To the frustration of firms and marketeers, opt-in rates on commercial websites are generally low. Options are usually presented to users as an opt-in or an opt-out structure. An opt-in is generally accepted as requiring express permission by an individual to allow the provision of some service or product, while an opt-out is where a course of action is pre-selected and the user must expressly deny that permission. The Cambridge Dictionary (Cambridge Dictionary, 2019) describes opt-in as "the fact of choosing to take part in an activity, arrangement, etc. rather than being forced to take part". The same source describes an opt-out as "to choose not to be part of an activity or to stop being involved in it." Consumers have become accustomed to dealing with numerous opt-in and opt-out services and charges as they negotiate micro-decisions in the transactional processes. In a paper describing certain design practices in the low cost carrier (LCC) sector, significant disquiet and exasperation were expressed by consumers in respect to the number and ambiguous nature of a series opt-in and opt-out services, many unavoidable, once they had committed to purchasing a flight (Barry et al., 2011a). 
IADIS International Journal on WWW/Internet

This paper describes a study of optionality focusing on how designers can frame decisions, the impact of default values, and the types of decision constructs. The analysis details the impact of each of these on errors in participant choice. Heat maps were also generated to illustrate where participants are looking on the screen and to what intensity. The findings reveal some exciting insights into the effect the presentation of choice can have on consumer outcomes. Other aspects of the findings, such as cognitive load and gender effects, will be reported upon in later work.

\section{CHOICE AND OPTIONALITY}

\subsection{Choice Framing}

The theory of rational choice, which has had such a tight hold over much research governing social and economic behaviour, has been questioned across a number of disciplines. Simon (1957) was among the first to signal its limitations by proposing the concept of 'bounded rationality'. Subsequently, Tversky and Kahneman (1981) theorised framed information could be encoded positively or negatively. Their research indicated the manner in which choice is framed to individuals significantly influences decision-making. They concluded "the dependence of preferences on the formulation of decision problems constitutes a significant concern for the theory of rational choice" Tversky and Kahneman (1981). Samuelson and Zeckhauser (1988) found decisions are often presented with 'influential labels', whereby there is nearly always one alternative that carries the label 'status quo'. In a series of experiments designed to test for status quo effects, they concluded decision-makers exhibited a significant choice bias towards the status quo. This also known as the default effect.

The default effect, a phenomenon whereby the user is more likely to choose the default option when presented with a choice, has been well documented in various studies (Johnson and Eagly, 1989; Ahmetoglu et al., 2010; Ploug et al., 2012; Anaraky et al., 2018). In the case of opt-outs, this can result in users making an inadvertent purchase or accidently signing up to a mailing list. In the case of an opt-in, the default option is to decline to purchase or to sign up to a mailing list, which may result in the user declining an option that may be of benefit to them. While there is research recognising opt-out decisions can be problematic, with users often inadvertently making a purchase, or opting in to a mailing list (Bellman et al., 2001), there is little research on negative consequences of errors with opt-ins in the transactional process. In fact, opt-ins are generally seen as the more benign option, with the EU requiring the use of opt-ins for all distance purchasing (European Union, 2011).

\subsection{Dimensions of Option Presentation}

The presentation of options to consumers in contemporary B2C interactions is made up of a number of dimensions. Much of the research discussed earlier on framing largely related to a singular dimension, that of a binary decision, choosing one of two options. In the more sophisticated world of online consumerism, the presentation of choice and optionality can be greatly finessed. The presentation of an option may now have multiple, even layered, dimensions. Previous research (Hogan et al., 2014; Torres et al., 2014) determined options tend to be presented to the user in a variety of ways. Some options are straightforward with easy to 
understand defaults and choices, while other options are more complicated and require effort to decipher so as to identify the default and the action required to achieve the desired outcome. In addition, some options are simply presented to the consumer while others incorporate various levels of persuasion, usually to encourage selection of the vendor's preferred outcome.

A desk analysis of 57 websites was conducted by the to determine the fundamental dimensions of option presentation for opt-out decision constructs in use in B2C websites (Barry et al., 2016). A total of 42 opt-outs were encountered across 17 of the websites examined. A number of dimensions were identified as contributing to option presentation: control type (e.g., checkbox, radio button or drop-down menu); default value (i.e., un-selected or pre-selected); question or information framing (i.e., acceptance, rejection or neutral language); general purpose of the construct (e.g., immediate revenue generation or permission to retain data); and additional persuaders (e.g., benefits of choosing the option or reassurance of privacy). In this study the default value and framing are the key variables examined. The work is restricted to checkboxes as these are the most commonly encountered control types for micro-decisions within the commercial transactional process.

\subsection{Option Framing}

Bellman et al. (2001) explored the impact of question framing on user decisions. In querying how consumers have unknowingly opted-in to something, they explored the tactics some firms employ to encourage consent. They identified different ways in which consent can be obtained and concluded there are consequential effects in how questions are presented to consumers. Indeed, by using the correct combination of question framing and default answers, firms 'can almost guarantee' consent. Lai and Hui (2006) also conducted research into the impact of question framing on user decisions. Their study indicates the manner in which the option is described, as well as the default option (i.e., checked or unchecked), has an impact on user choice. They found that for opt-in decisions using checkboxes, users are more likely to accept an un-selected opt-in over a pre-selected opt-in. They suggest the positive language of acceptance is likely to influence the users' decision.

Question framing has also been shown to affect user choice (Tversky and Kahneman, 1981; Levin et al., 1998; Kuo et al., 2009; Anaraky et al., 2018), with positively framed messages more likely to result in the user opting in to the choice. However, most of the studies presented the choice to the user in a way that either emphasized the positive or negative consequences of accepting or rejecting the choice. Lai and Hui (2004) did examine the impact of acceptance and rejection framing as defined in this paper, along with the impact of the default value (either pre-selected or un-selected). They determined opt-ins were more likely to result in a positive selection when acceptance framing was coupled with an un-selected presentation. They identified no significant difference for opt-outs.

\subsection{Using Opt-out Structures for Altruistic Purposes}

While there are obvious privacy concerns in respect of the use by firms and governments of personal data for purposes that do not serve the individual well, there are also cogent arguments for the use of opt-out structures to persuade or nudge people into making positive choices. These might include promoting the consumption of better foods like whole wheat (Van Kleef et al., 2018), renewable energy (Momsen and Stoerk, 2014), organ donation (Johnson and Goldstein, 
IADIS International Journal on WWW/Internet

2003; Van Dalen and Henkens, 2014), climate change (Ebeling and Lotz, 2015), green environment (Schubert 2017), environmental conservation in hotels (Ebeling and Lotz, 2015), health surveys (Johnson et al., 2002) and retirement savings (Madrian and Shea, 2001; Beshears et al., 2009).

\subsection{Using Opt-out Structures for Self-serving Outcomes}

Some marketing literature make no apology for the use of opt-out structures to upsell or distribute newsletters, while others claim that consumers are not always well-served by opt-in restrictions. For example, recent research reported "how travel companies can benefit from default options to promote the sale of higher-graded - and thus higher-priced - travel packages" (Steffen et al., 2019). McQuinn has suggested that "if companies are forced to live with opt-in rules, the higher costs involved would ultimately be passed along to consumers in the form of higher prices, or would result in fewer free services" (McQuinn, 2017). In that article it was reported that one U.S. firm, Qwest, who conducted their own research determined that it would simply not be viable to use an opt-in strategy, citing the cost of persuading customers to opt-in.

In the context of online, transactional processes rather than data privacy or altruistic motivations, it has been observed that in recent years that some firms in particular sectors such as airlines, online car hire firms and hotel comparison sites have been using design techniques that are not just unorthodox but of apparent deliberate design with a variety of self-serving ambitions. These include: making options unclear or ambiguous, leading consumers to make an unnecessary choice; or deconstructing a final price with taxes, charges and items presented as options but which are, in fact, essential elements of the product or service (Barry et al., 2011; Hogan et al., 2014).

\subsection{Arguments for the Use of Opt-in Structures}

While the United States and the European Union agree on the importance of enhancing privacy protection (as evidenced by the EU-U.S. Privacy Shield Framework), they differ in their approach to privacy and the framing of certain consumer online interactions. Broadly, the United States favours self-regulation and opt-out framing, whilst the European Union prefers opt-in framing (European Union, 2008).

Issues in respect of questionable practices being used by European airlines, especially low-cost carriers, led to a European Commission investigations into airline ticket selling. It was conducted under the auspices of Consumer Protection Co-operation Regulation, which came into force in 2007 (EEC-Net, 2007). The report identified unfair practices related to the availability of special offers, price indications and contract terms. The Commission directed airlines to give consumers the total price, including taxes and booking/credit card fees, in the first advertised price when interacting online. Other unfair practices include the mandatory purchase of insurance, or presenting optional services such as insurance or priority boarding as opt-outs. Previously, The European legislation governing airlines' price information was laid out in Articles 5-7 of the Unfair Commercial Practices Directive (European Union, 2005). 


\section{RESEARCH DEVELOPMENT}

\subsection{Eye Tracking Research}

Eye tracking has been widely used in HCI and web usability research, as well as in many other fields such as marketing and psychology (Di Stasi et al., 2011; Djamasbi et al., 2011; Huang and Kuo, 2011). By following the gaze of users as they interact with a web page, it is possible to capture data on the areas of the screen on which they concentrate their interest and to determine eye movement patterns. This data may then be analysed to draw inferences on user decision-making strategies (Day et al., 2006), (Glöckner and Herbold, 2011), (Huang and Kuo, 2011). While previously qualitative studies have been conducted to capture user intentions and understandings, the potential of gathering hard, physiological data about participant behaviour in interactive decision-making was a key motivation for developing an Eye Tracking Laboratory at the authors' university. Coupled with the soft data that may be captured from users describing what they see and experience, eye tracking data contribute objective measurements of the visual pattern of the interaction and provide a much richer and nuanced understanding of the user's approach to decision making.

The eye tracking technology system used in this study involves the projection of an infrared light and a video camera on a person's eye to identify where they are looking on a screen (Nielsen and Pernice, 2010). Eye movement patterns on webpages are much more erratic than one might anticipate. When users pause to concentrate on an area of interest, they fix their gaze on it, and it then comes into sharp focus. If a user's gaze remains fixed for more than 3 milliseconds this becomes a fixation, while the movements in-between fixations are known as saccades and convey no visual input. All eye movements are recorded by the eye tracking software. Examining the number and duration of the fixations, as well as the pattern of movement across the screen provides valuable information on the user's understanding of what they are seeing.

\subsection{Preparation for the Study}

The authors expected the development of an eye tracking experiment to study decision-making in an online transactional process would be challenging and risky, more so given they had no previous eye tracking experience and the laboratory was newly established. Thus, it was decided to first design and run a pilot study to validate the research design. The purpose was to learn lessons from the process of constructing an eye tracking experiment and to fine-tune the research instruments (Hogan et al., 2015).

Eye tracking and Cued Retrospective Think Aloud (RTA) were used to investigate potentially problematic decision constructs. The laboratory-based, interactive study examined how certain decision constructs impacted upon users' decision-making and their cognitive processes. The main lessons learned were to ensure participants are fully briefed before commencing the test; that they perform tasks, as instructed, and working at their normal pace; and are de-briefed immediately after the experiment to reveal insights into their behaviour. The key contributions of the study were the identification of improvements to be made to the research design, robust experiment administration and the refinement of the research instruments. 


\subsection{Research Approach}

As noted in Barry et al. (2016), eye tracking technology has been used extensively in web usability studies, often alongside think-aloud techniques. This research project was conducted in two parts. In the first set of experiments on opt-out decision constructs, data was collected from 114 participants, 456 experiment trials and 23 Cued Retrospective Think-Aloud (RTA) sessions. The second set concerned opt-in decision constructs and collected data from 51 participants, 204 experiment trials and 12 Cued RTA sessions. The project was designed like this to control for construct type bias and potential confusion. This design was informed by experiences with the earlier pilot study. Thus statistical analysis was conducted, where relevant, on a within-subjects or between-subjects basis. The study was made up of three dimensions: an eye tracking experiment; self-assessment evaluations (on subjective cognitive load); and Cued RTA sessions.

\subsection{The Eye Tracking Experiment}

As the pilot study had highlighted the risks associated with the use of the eye tracking technology, significant effort was spent planning the experiments. Each of the variants of opt-out and opt-in decisions were examined (Hogan et al., 2014): Un-selected Rejection Framing; Pre-selected Acceptance Framing; Pre-selected Rejection Framing; Pre-selected Neutral Framing; Un-Selected Acceptance Framing; and Un-Selected Neutral Framing (see Table 1).

Table 1. Decision constructs types

\begin{tabular}{lcccc}
\hline Construct Name & $\begin{array}{c}\text { Construct } \\
\text { Type }\end{array}$ & Default Value & Framing & Presented In \\
\hline Un-Selected Rejection & USR & Un-selected & Rejection & Opt-in and Opt-out \\
\hline Pre-Selected Acceptance & PSA & Pre-selected & Acceptance & Opt-in and Opt-out \\
\hline Pre-Selected Rejection & PSR & Pre-selected & Rejection & Opt-out \\
\hline Pre-Selected Neutral & PSN & Pre-selected & Neutral & Opt-out \\
\hline Un-Selected Acceptance & USA & Un-selected & Acceptance & Opt-in \\
\hline Un-Selected Neutral & USN & Un-selected & Neutral & Opt-in \\
\hline
\end{tabular}

The opt-out and opt-in decisions presented to participants were similar to real-world micro-decisions ordinarily facing users when engaged in a commercial transactional process on websites (Hogan et al., 2014; Barry et al., 2016). In each study, participants were presented with either four opt-in or four opt-out micro-decisions. Participants were told to buy the product in two instances and not to buy in the other two. For opt-in decisions, the participant needed to take action to make a purchase. Thus, the default option was to decline the purchase. For opt-out decisions, the participant needed to take action to decline the purchase. Thus, the default option being to purchase the item. The order of presentation of the four screens was randomized when presented to participants. The core webpage screen was a breakdown insurance product to which participants were asked if they require an enhanced monthly-costed, add-on feature. Each screen had a decision point with a checkbox beside it (see Table 2). 
Table 2. Decision constructs presented to participants

\begin{tabular}{ll}
\hline $\begin{array}{l}\text { Construct } \\
\text { Type }\end{array}$ & \multicolumn{1}{c}{ Decision Construct* } \\
\hline PSA opt-out & $\square$ Rescue Plus includes free car hire and travel expenses. I want to purchase Rescue Plus. \\
\hline PSN opt-out & $\square$ Rescue Plus. \\
\hline PSR opt-out & $\square$ Rescue Plus includes free car hire and travel expenses. If you would rather not purchase Rescue Plus, please untick this box. \\
\hline USR opt-out & $\square^{\text {Rescue Plus includes free car hire and travel expenses. If you would rather not purchase Rescue Plus, please tick this box. }}$ \\
\hline USA opt-in & $\square^{\text {Rescue Plus includes free car hire and travel expenses. I want to purchase Rescue Plus. }}$ \\
\hline USN opt-in & $\square$ Rescue Plus. \\
\hline USR opt-in & $\square$ Rescue Plus includes free car hire and travel expenses. If you would rather not purchase Rescue Plus, please leave this box unticked. \\
\hline PSA opt-in & $\square$ Rescue Plus includes free car hire and travel expenses. If you would like to purchase Rescue Plus, please untick this box. \\
\hline
\end{tabular}

* The decision constructs are illustrated as they appeared on the screens

\section{EXPLORATORY ANALYSIS}

Once the data was gathered, exploratory analysis was conducted. The main focus of this analysis was participant error rates. A participant is deemed to have made an error when they make a mistake in the decision they have been told to take. For example, when told to 'Buy Rescue Plus Insurance' by choosing to tick or untick a checkbox, they unknowingly take the incorrect action. The error rates for opt-in and opt-out constructs were compared and contrasted. Framing and default value were also examined.

\subsection{Overall Error Rate}

The first step in exploring our data was to examine the error rate for the different construct types.

Table 3. Error rate for opt-out constructs

\begin{tabular}{cccc}
\hline Construct Type & Correct & Incorrect & Total \\
\hline PSA & $94(82 \%)$ & $20(18 \%)$ & 114 \\
\hline PSN & $87(76 \%)$ & $27(24 \%)$ & 114 \\
\hline PSR & $86(75 \%)$ & $28(25 \%)$ & 114 \\
\hline USR & $72(63 \%)$ & $42(37 \%)$ & 114 \\
\hline Total & $339(74 \%)$ & $117(26 \%)$ & 456 \\
\hline
\end{tabular}

For opt-outs (see Table 3) the number of incorrect selections varied between the construct types, with PSA having the highest rate of correct selections and USR having the highest rate of incorrect selections. Overall, the rate of incorrect selections made by participants was high, ranging from $18 \%$ (PSA) to $37 \%$ (USR). Chi-square test was conducted to determine whether 
IADIS International Journal on WWW/Internet

the relationship between construct type and error rate was significant. The test indicated significance $\left(\chi^{2}(3, \mathrm{~N}=456)=11.715, \mathrm{p}<0.01\right)$. However, the value for $\phi$ was 0.158 , indicating the association was weak, only accounting for $2.5 \%$ of the variation.

Table 4. Error rate for opt-in constructs

\begin{tabular}{cccc}
\hline Construct Type & Correct & Incorrect & Total \\
\hline USN & $43(84 \%)$ & $8(16 \%)$ & 51 \\
\hline USA & $41(80 \%)$ & $10(20 \%)$ & 51 \\
\hline USR & $36(71 \%)$ & $15(29 \%)$ & 51 \\
\hline PSA & $25(49 \%)$ & $26(51 \%)$ & 51 \\
\hline Total & $145(71 \%)$ & $59(29 \%)$ & 204 \\
\hline
\end{tabular}

For opt-ins (see Table 4) the number of incorrect selections also varied between the construct types, with PSA having the highest incorrect rate and USN having the highest correct rate. Overall, the rate of incorrect selections made by participants was high, ranging from $16 \%$ (USN) to $51 \%$ (PSA). Chi-square test was conducted to determine if the relationship between construct type and error rate was significant. The test indicated significance $\left(\chi^{2}(3, \mathrm{~N}=204)=18.576\right.$, $\mathrm{p}<0.01)$. However, the value for $\phi$ was 0.302 , indicating the association was relatively weak, only accounting for $9.1 \%$ of the variation. The error rate for PSA was almost double that of the next highest, suggesting this construct was particularly problematic.

\subsection{Opt-in versus Opt-out Error Rates}

As can be seen in Tables 3 and 4 above, the error rate was high for both opt-in and opt-out constructs, with $26 \%$ of decisions on opt-out constructs incorrect, and $29 \%$ of decisions on opt-in constructs incorrect. The variation in error rates is wider for opt-ins (16-51\%) than for opt-outs (18-37\%). The two opt-in constructs with the highest error rates (PSA and USR) are rarely encountered in practice (Hogan et al., 2014), with most opt-ins encountered being the more straightforward USA or USN. This is perhaps due to the lack of benefit to the seller associated with misinterpretation of an opt-in. With the opt-outs, the USR has a considerably higher error rate than the other constructs. Again, this construct type is less frequently encountered than the others (Hogan et al., 2014; Barry et al., 2016). However, it is still regularly encountered, particularly in sign-ups for mailing lists. The higher prevalence of this opt-out construct may be due to the perceived advantages to firms associated with users selecting the option, whether intentionally or erroneously.

The error rate for opt-in and opt-out were compared using Chi-square. Opt-in had 71\% correct and $29 \%$ incorrect while opt-out had $74 \%$ correct and $26 \%$ incorrect. No significant difference was seen between the opt-in and opt-out constructs. As the USR for opt-out and the PSA for opt-in are rarely encountered, the error rates were compared for the more commonly encountered constructs. Again, there was no significant difference detected. Previous research (Bellman et al., 2001; Lai and Hui, 2006; Ahmetoglu et al., 2010) has reported increased likelihood of users signing up or purchasing when presented with an opt-out, rather than an opt-in, finding framing and default value significantly impact user decisions. The findings in this study, do not support that premise (a similar result was found by Abbink and Hennig-Schmidt (2006)), perhaps because participants had pre-decided their desired outcome rather than being undecided. 


\subsection{The Effect of Default Action on Error Rate}

The data was analyzed to determine whether the default action impacted on participants accuracy when making micro-decisions. For the opt-out constructs, the default action was to buy the add-on whereas for the opt-in constructs, the default action was to not buy the add-on. Chi-square was used to determine whether there was a significant difference in the error rate, depending on whether the participant's instruction was to choose the default action for the construct or to take action to change the default action. The analysis indicated no significant difference in error rates $\left(\chi^{2}(1, \mathrm{~N}=204)=0.024, \mathrm{p}=0.877\right.$ for opt-in and $\chi^{2}(1, \mathrm{~N}=456)=2.587$, $\mathrm{p}=0.108$ for opt-out). This contradicts previous research indicating users tend to choose the default action when presented with a choice. However, in previous research (Johnson and Eagly, 1989; Ahmetoglu et al., 2010; Ploug et al., 2012; Anaraky et al., 2018) participants were allowed a free choice when presented with the options. The studies either examined rates of acceptance of the default action or presented the participants with a free choice. In this study, participants were told to buy or not buy so they had already made their decision regarding which action they would take. This suggests the default action only affects choice when the user has not already decided which action they wish to take. Therefore, the default effect can be seen as an influencer for the undecided user, rather than a persuader for a change of mind.

\subsection{The Effect of Construct Design on Error Rate}

In this study the options were presented to participants using three different types of framing, as identified by the authors in previous research (Hogan et al., 2014). The frames (acceptance, rejection and neutral framing) are all commonly used in the presentation of micro-decisions on websites. Additionally, this study examines error rate when the user has pre-decided their course of action, rather than being undecided in their choice.

Lai and Hui (2004) suggest that the tick in a pre-selected checkbox can have an anchoring effect on users which results in amplification of the framing. Thus, a pre-selected checkbox with rejection framing acts as a negative anchor and is more likely to result in the user rejecting the option while a pre-selected checkbox with acceptance framing acts as a positive anchor and is more likely to result in the user accepting the option. If this were the case, one might see more errors for participants instructed to not buy than those instructed to buy for the PSA constructs and to see more errors for those instructed to buy than those instructed to not buy for PSR constructs. No significant difference was seen for either PSA or PSR when Chi-square tests were run. This suggests that while an anchoring effect may nudge a user towards a particular decision, it will not cause them to mistakenly choose an option.

\subsection{The Effect of Framing on Error Rate}

As default actions can impact on the users' selection, the framing of opt-in and opt-out constructs was examined separately as they have different default actions. For opt-out, acceptance framing had the highest rate of correct selections, followed by neutral framing, with rejection framing having the highest rate of incorrect selections (see Table 5). A Chi-square test indicated that the framing had a significant effect on the error rate $\left(\chi^{2}(2, \mathrm{~N}=456)=7.209\right.$, $\mathrm{p}=0.027)$. However, the value for $\phi$ was 0.126 , indicating the association was weak, only accounting for $1.6 \%$ of the variation. Although the anchor effect did not have a significant effect 
IADIS International Journal on WWW/Internet

on the error rate, the one un-selected construct type was eliminated from the test as it may have impacted on the result when coupled with framing. No significant difference was seen, suggesting that framing on its own does not impact on error rate for opt-out constructs.

Table 5. Error rate for opt-out constructs

\begin{tabular}{lccc}
\hline Framing & Correct & Incorrect & Total \\
\hline Acceptance & $94(82 \%)$ & $20(18 \%)$ & 114 \\
\hline Neutral & $87(76 \%)$ & $27(24 \%)$ & 114 \\
\hline Rejection & $158(69 \%)$ & $70(31 \%)$ & 228 \\
\hline Total & $339(74 \%)$ & $117(26 \%)$ & 456 \\
\hline
\end{tabular}

The tests were repeated for opt-ins (see Table 6). When all constructs were considered, a significant difference was seen $\left(\chi^{2}(2, \mathrm{~N}=204)=6.637, \mathrm{p}=0.041\right)$. However, the value for $\phi$ was 0.177 , a weak association, only accounting for $3.1 \%$ of the variation. The test was repeated using only the un-selected opt-ins. No significant difference was seen, suggesting framing on its own does not impact error rate for opt-in constructs.

Table 6. Error rate for opt-in constructs

\begin{tabular}{lccc}
\hline Framing & Correct & Incorrect & Total \\
\hline Acceptance & $66(65 \%)$ & $36(35 \%)$ & 102 \\
\hline Neutral & $43(84 \%)$ & $8(16 \%)$ & 51 \\
\hline Rejection & $36(71 \%)$ & $15(29 \%)$ & 51 \\
\hline Total & $145(71 \%)$ & $59(29 \%)$ & 204 \\
\hline
\end{tabular}

\section{HEAT MAPS}

To gain additional understanding of the error rate encountered, eye tracking heat maps were examined to explore where participants focused their attention during the experiment. Heat maps visually show where the viewer focuses their attention during an interaction. The more time spent focusing on a segment of the screen, the 'hotter' the colour. The areas receiving the most attention are coloured red, those with less attention, orange and those with less again are coloured green. Areas that receive no attention are not coloured. The heat maps were broken down by construct type and then into those who made the correct selection and those who made the incorrect selection.

\subsection{Opt-out Heat Maps}

For the opt-outs, the construct with the highest rate of error (37\%) was the un-selected rejection (USR) construct. As can be seen in Figure 1, participants who made the correct decision exhibited some pronounced gaze, and presumably reading, intensity along the construct text. The heat map is considerably redder around the words would rather not purchase and please tick for those who got it correct. 


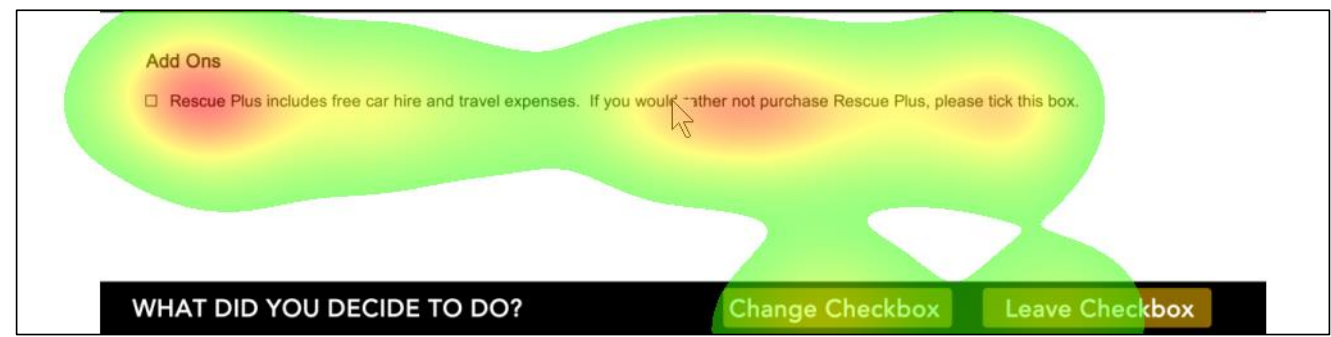

Figure 1. Un-selected rejection framing - opt-out - correct selection

In contrast, Figure 2 illustrates that participants who made the incorrect decision for the USR were less likely to read the text which explains, if somewhat cryptically, the correct action to take if they did not wish to purchase Rescue Plus. It is clear that those who made the incorrect decision paid less attention to the wording of the construct, relying instead on the pre-selected or un-selected state of the checkbox. It is safe to conclude that the un-selected checkbox may have suggested the decision construct was an opt-in rather than an opt-out, thus resulting in the high error rate. This is confirmed when heats maps for individual participants were examined.

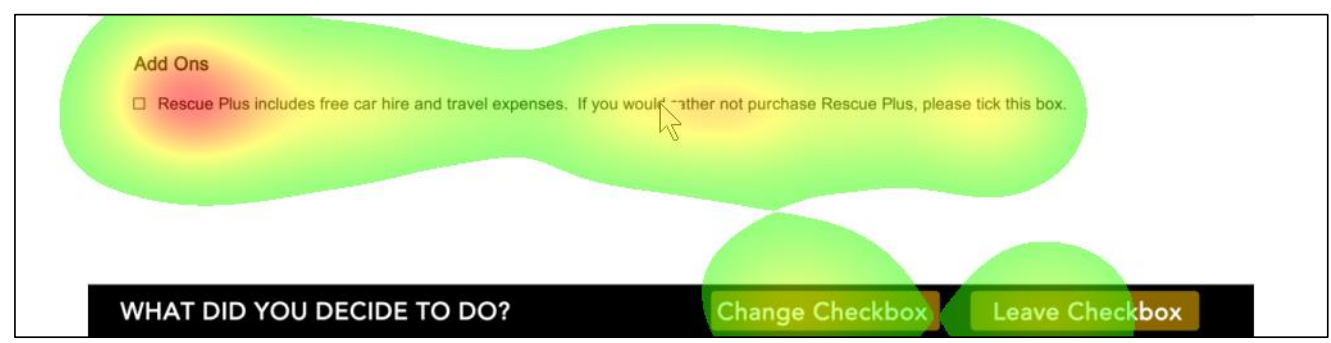

Figure 2. Un-selected rejection framing - opt-out - incorrect selection

Participants were instructed to interact with the decision constructs in the way they would normally do. As can be seen in Figure 3 some individuals only looked at the checkbox and did not read any of the text in the decision construct and so made their decision solely on the presentation of the checkbox. In reality, this behaviour is not unexpected as users quickly deal with multiple micro-decisions along the transactional process to reach the conclusion of the interaction.

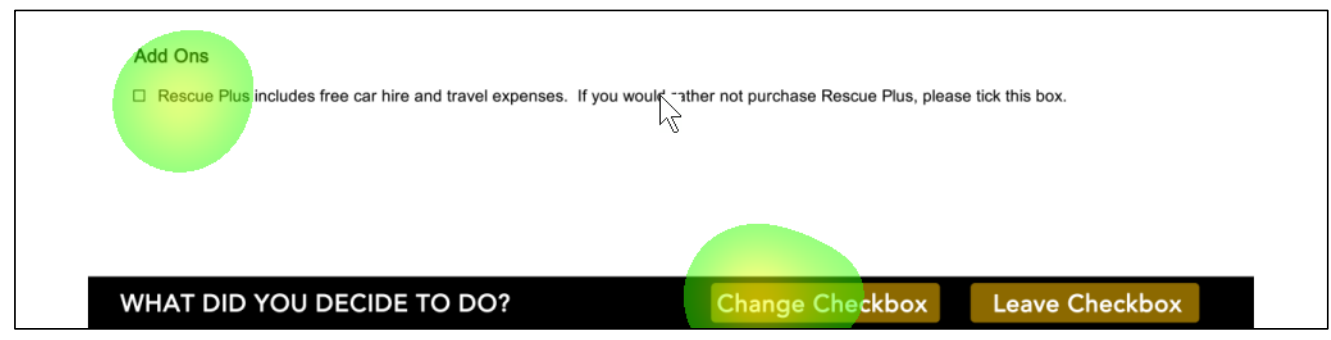

Figure 3. Un-selected rejection framing - opt-out - incorrect selection for individual participant 
IADIS International Journal on WWW/Internet

The opt-out construct with the least number of errors was the Pre-selected Acceptance (PSA). The heat maps, Figure 4 and Figure 5, show no discernible difference between those who made the correct selection and those who got it wrong. However, in both maps, the major focus was on the checkbox rather than the text giving instruction. This shows that participants paid considerably less attention to the text than to the checkbox, suggesting that, for many, their decision on the type of construct was predominantly determined by the checkbox. In this case, the pre-selected nature of the checkbox appears to have suggested to the participant that the construct was an opt-out, hence the lower error rate.

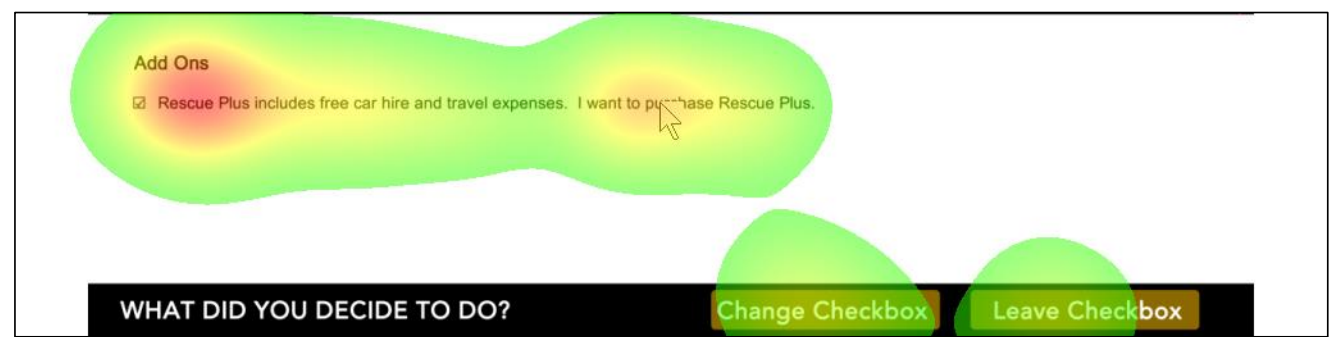

Figure 4. Pre-selected acceptance framing - opt-out - correct selection

The level of focus on the instructions in the incorrect PSA (Figure 5) is similar to that on the instructions for those who made an error on USR. However, the pre-selection on the checkbox for PSA suggests an opt-out and therefore the participants were less likely to make an error.

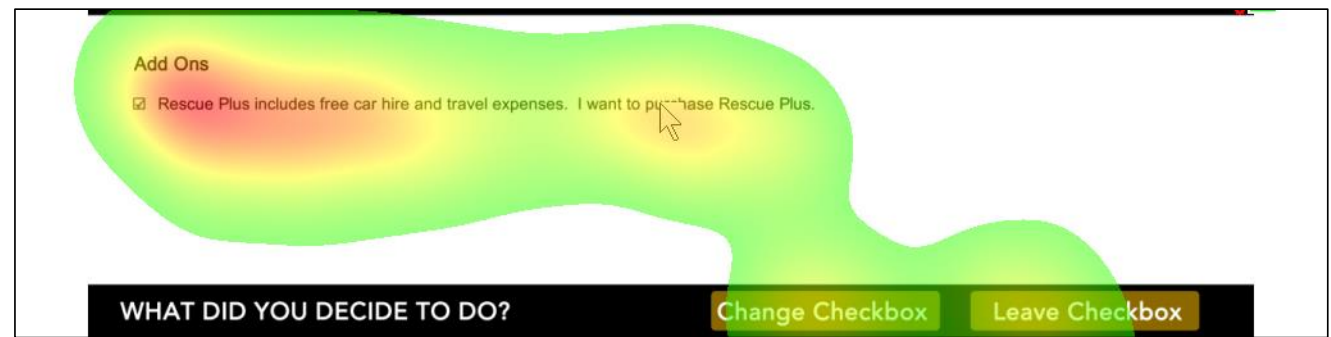

Figure 5. Pre-selected Acceptance Framing - Opt-out - Incorrect Selection

A similar pattern to USR was found in PSR. Participants who made an error were less likely to focus on the instructions. However, the pre-selection 'suggests' an opt-out and so, the error rate was significantly lower than for the USR. However, the error rate for PSR was higher than for PSA. It is possible the framing led to some confusion on the part of the participants who read the instructions and they then made the wrong choice. The error rate for PSN was similar to that of PSR. However, there is no indication from the heat maps as to why the error rate was so high (24\%). This will be explored in further work when the cued RTAs are analysed. This analysis will likely provide insight into the reasoning of participants who made the incorrect choice with PSNs. 


\subsection{Opt-in Heat Maps}

For the opt-ins, the construct with the highest rate of error (51\%) had pre-selected acceptance (PSA) framing. As can be seen in Figure 6, the heat map for those who made the correct decision exhibits some redness around the words want to purchase and please untick, indicative of reading. Nonetheless, far more attention remains focused around the checkbox.

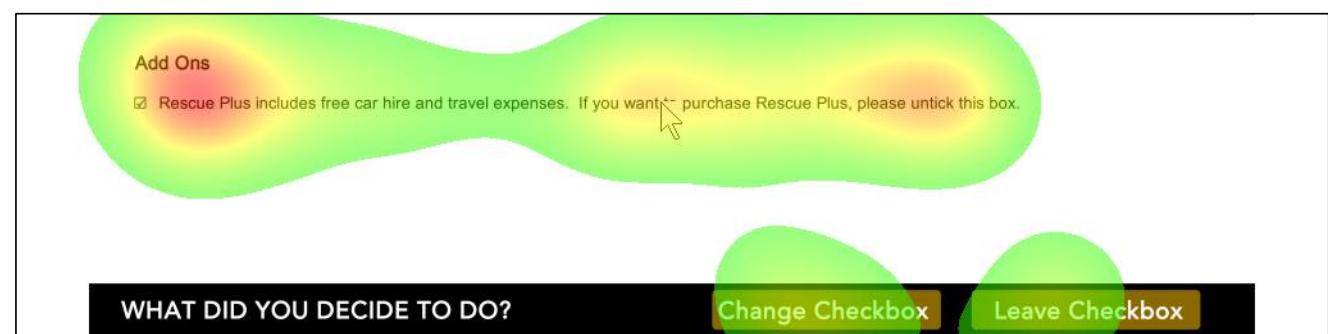

Figure 6. Pre-selected acceptance framing - opt-in - correct selection

Figure 7 illustrates that participants who made the incorrect PSA decision were less likely to read the text that indicates the action required to purchase Rescue Plus. These participants paid far less attention to the wording of the critical second sentence. Their focus and decision was primarily based on whether the checkbox was un-selected or pre-selected. An obvious interpretation was that the pre-selected checkbox was suggestive of an opt-out rather than an opt-in construct, resulting in the high error rate. Since the great majority of opt-ins encountered in practice are un-selected, many participants were likely to have been predisposed to this assumption. As with the opt-out, when individual participants' interactions were examined, many paid little attention to the text, relying instead on the suggestive status of the checkbox and their a priori expectations.

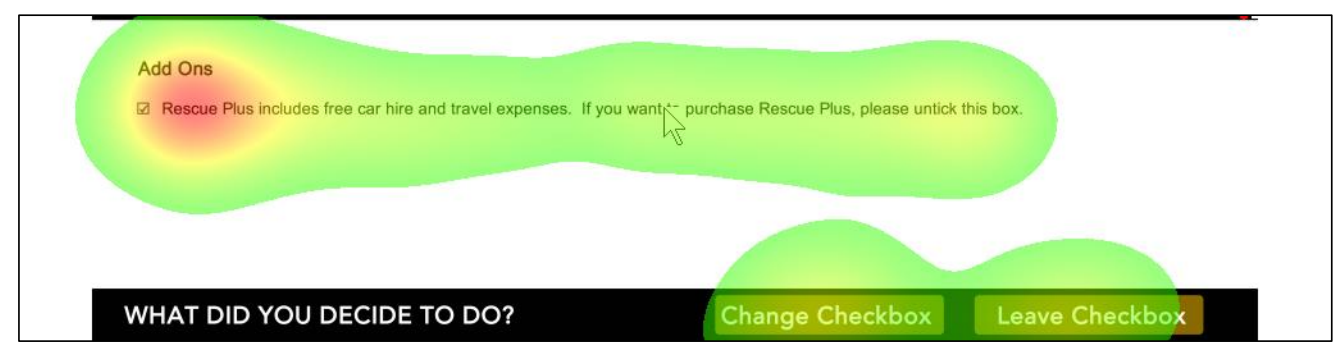

Figure 7. Pre-selected acceptance framing - opt-in - incorrect selection

When examining the heat maps for the opt-in construct (USN) which had the least number of errors (see Figure 8 and Figure 9), there is no discernible difference between them in respect to participant gaze on the decision construct. However, as it is neutral framing (neither persuasive nor indicative), there is no instructional text for the participant to read. However, those who made the incorrect decision spent less time deliberating whether to change or leave the checkbox. This is illustrated by the level of 'heat' on the change and leave buttons. 


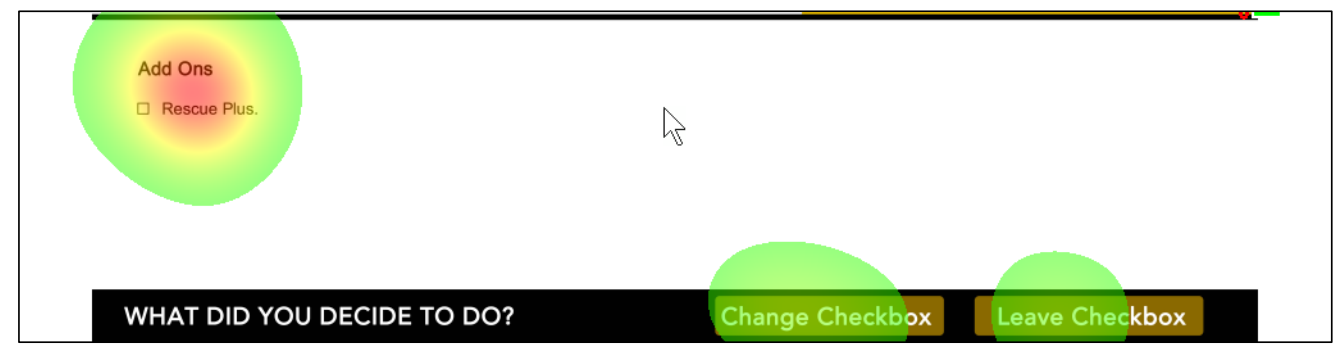

Figure 8. Un-selected neutral framing - opt-in - correct selection

In the case of all neutral constructs, both opt-in and opt-out, there was no major difference between the level of focus on the checkbox part of the construct between those who made an error and those who did not.

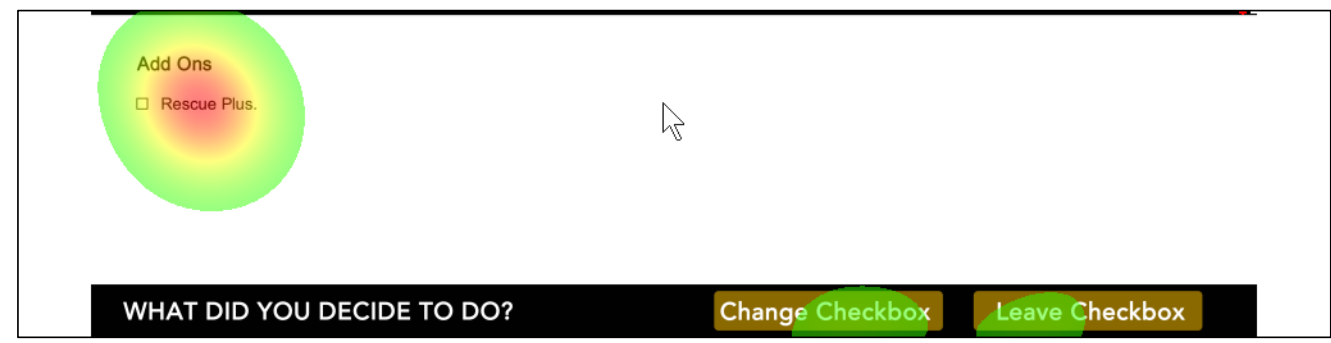

Figure 9. Un-selected neutral framing - opt-in - incorrect selection

In the case of the USA opt-in construct (error rate of 20\%) there was little difference in the heat map pattern between those making the correct decision compared with those in error. However, for the USR (error rate 29\%), those who made an incorrect decision were again less likely to have read the instructional part of the construct and therefore more likely to use the checkbox alone to determine the type of construct they were dealing with.

\section{CONCLUSIONS}

This exploratory study set out to examine how the design of constructs used for online, commercial transactions impacted on error rates for opt-out and opt-in micro-decisions. The error rate was remarkedly high for all construct types, some considerably higher than others. The PSA for opt-in had an error rate $(51 \%)$ of over three times the lowest error rate for opt-in ( $16 \%$ for USN), suggesting it was hugely misleading. It is unusual, even unlikely, to encounter a pre-selected opt-in on a website, and participants appear to have assumed a pre-selected checkbox was an opt-out. Since, in practice, the use of the PSA opt-in construct would not be in the interest of firms, the overall opt-in error rate is skewed by its inclusion. Excluding the construct reveals an overall opt-in error rate of $22 \%$, lower than that of the opt-out average of $26 \%$. Nonetheless, no significant difference was found, suggesting users are no more likely to erroneously select an opt-out than an opt-in. 
The default effect is a phenomenon whereby users, when presented with options, are more likely to accept the default option than change it. In this study, participants were told what decision to make when presented with the construct. If the default effect significantly impacted the outcome, a higher error rate would be expected when the participant was required to change from the default action. No significant difference was seen between the error rates, suggesting the default effect does not apply when the user has already decided on the course of action they wish to take. Therefore, while it may influence the undecided user, it is unlikely to dissuade the decided user from their planned course of action.

The anchoring effect of a pre-selected checkbox has been shown previously to reinforce the framing of a construct, whereby acceptance framing is more likely to result in option acceptance and rejection framing is more likely to result in option rejection. It was posited that this results from more time spent contemplating the text presented to the user. In this study, no anchoring effect was apparent, likely because participants had pre-decided on the course of action and so, were less susceptible to the nudge power of checkbox pre-selection. Once the default value of the decision construct was controlled, the framing of the construct did not have a significant effect on the error rate for either opt-in or opt-out.

The findings in this study differ from previous ones with respect to framing, anchor and default effect directing users to a particular course of action. The fact the participants had predetermined the outcome is likely to have negated the framing, default and anchor effects. In addition this study, in contrast to other studies, has examined all of the various opt-in and opt-out construct types, suggesting the effects are much more nuanced than previously thought.

In order to shed some light on the high error rate, heat maps were examined. Heat maps illustrated the areas of the constructs participants focused on during their interactions. It would appear that many of the participants who made an incorrect decision, only used the checkbox to determine whether the decision construct was an opt-in or an opt-out and focused less on the instructional text. It seems a pre-selected checkbox suggested opt-out while an un-selected checkbox suggested opt-in. Obviously, some participants still made an incorrect choice even when they did read the instructional text but the heat maps demonstrate that they spent considerably less time reading the explanatory text than those who did not make a mistake. This strongly suggests users frequently make assumptions based on partial information. They simply glance at the construct, see a checkbox and decide what to do based on whether the checkbox is unselected or pre-selected.

This study has added to the body of knowledge on the use of opt-in and opt-out constructs for micro-decisions on commercial websites. Previous research suggests designing constructs in a particular way can increase the number of users accepting or rejecting the option presented. This study clearly indicates that the user's interaction with micro-decisions is considerably more complex than previously thought. Many users make quick, uninformed decisions based on a visual interpretation of the construct, rather than examining or reading the text in any detail.

In order to gain further insight into users' thought process and mental reasoning when interacting with micro-decisions, the next phase of this research will examine the data from the cued RTAs and a deconstruction of the micro-decision into multiple interest areas. The cued RTA process involved showing the participants an animation of their visual interaction and asking them to articulate their understanding and the thought process they followed whilst interacting with the micro-decisions. A preliminary examination of this data supports our supposition that users rely on the visual elements of the constructs in order to determine whether it is an opt-out or and opt-in. We will also break down the checkbox and instructional text sentences into separate interest areas and test for correlations between error rates and the extent to which participants actually read the text. 
IADIS International Journal on WWW/Internet

\section{REFERENCES}

Abbink, K. and Hennig-Schmidt, H., 2006. Neutral versus loaded instructions in a bribery experiment. Experimental Economics, Vol. 9, No. 2, pp. 103-121.

Ahmetoglu, G. et al, 2010. Pricing Practices: Their Effects on Consumer Behaviour and Welfare. Mountainview Learning. University College London.

Anaraky, A. et al, 2018. Reducing Default and Framing Effects in Privacy Decision-Making. SIGHCI 2018 Proceedings.

Barry, C. et al, 2011. Low-Cost Carriers and High-Tech Barriers - User Views on Questionable Web Design Practices in Ireland. Irish Journal of Management, Vol. 31, No. 1, pp. 43-58.

Barry, C. et al, 2011. Perceptions of Low Cost Carriers' Compliance with EU Legislation on Optional Extras. 20th International Conference on Information Systems Development. Edinburgh, Scotland,

Barry, C. et al, 2016. Framing or Gaming? Constructing a Study to Explore the Impact of Option Presentation on Consumers. Information System Development: Transforming Healthcare Through Information Systems, Springer International Publishing: 111-124.

Bellman, S. et al, 2001. On site: to opt-in or opt-out?: it depends on the question. Communications of the $A C M$, Vol. 44, No. 2, pp. 25-27.

Beshears, J. et al, 2009. The importance of default options for retirement saving outcomes: Evidence from the United States. Social security policy in a changing environment, University of Chicago Press: 167-195.

Cambridge Dictionary, 2019. Opt-in.

Day, R. F. et al, 2006. The effect of flash banners on multiattribute decision making: distractor or source of arousal? Psychology \& Marketing, Vol. 23, No. 5, pp. 369-382.

Di Stasi, L. L. et al, 2011. Main sequence: an index for detecting mental workload variation in complex tasks. Applied ergonomics, Vol. 42, No. 6, pp. 807-813.

Djamasbi, S. et al, 2011. Online viewing and aesthetic preferences of generation Y and the baby boom generation: testing user web site experience through eye tracking. International Journal of Electronic Commerce, Vol. 15, No. 4, pp. 121-158.

Ebeling, F. and Lotz, S., 2015. Domestic uptake of green energy promoted by opt-out tariffs. Nature Climate Change, Vol. 5, No., pp. 868.

EEC-Net, 2007. Report on Air Passenger Rights: Consumer Complaints 2006. European Consumer Centre Network, European Union.

European Union, 2005. Unfair Commercial Practices Directive

European Union, 2008. Regulation of the European Parliament and of the Council on Common Rules for the Operation of Air Services in the Community (recast)

European Union, 2011. Directive on Consumer Rights

Glöckner, A. and Herbold, A. K., 2011. An eye-tracking study on information processing in risky decisions: Evidence for compensatory strategies based on automatic processes. Journal of Behavioral Decision Making, Vol. 24, No. 1, pp. 71-98.

Hogan, M. et al, 2014. Theorising and testing a taxonomy of decision constructs. Journal of Customer Behaviour, Vol. 13, No. 3, pp. 171-185.

Hogan, M. et al, 2015. An Eye Tracking Pilot Study on Optionality - Some Lessons Learned. 17th Irish Academy of Management Annual Conference. Galway,

Huang, Y. and Kuo, F., 2011. An eye-tracking investigation of internet consumers' decision deliberateness. Internet Research, Vol. 21, No. 5, pp. 541-561.

Johnson, B. T. and Eagly, A. H., 1989. Effects of involvement on persuasion: A meta-analysis. Psychological Bulletin, Vol. 106, No. 2, pp. 290-314. 
Johnson, E. J. et al, 2002. Defaults, Framing and Privacy: Why Opting In-Opting Out1. Marketing Letters, Vol. 13, No. 1, pp. 5-15.

Johnson, E. J. and Goldstein, D. G., 2003. Do defaults save lives? Science, Vol. 302, No., pp. 1338-1339.

Kuo, F. et al, 2009. An exploratory study of cognitive effort involved in decision under Framing - an application of the eye-tracking technology. Decision Support Systems, Vol. 48, No. 1, pp. 81-91.

Lai, Y. and Hui, K., 2004. Luring the Prey: Attracting Online Consumer Participation through Choice Configurations. American Market Association (AMA) Marketing and Public Policy Conference. Washington D.C., USA,

Lai, Y. and Hui, K., 2006. Internet opt-in and opt-out: investigating the roles of frames, defaults and privacy concerns. Proceedings of the 2006 ACM SIGMIS CPR conference on computer personnel research: Forty four years of computer personnel research: achievements, challenges \& the future. Claremount, California, USA, 253-2631.

Levin, I. P. et al, 1998. All frames are not created equal: A typology and critical analysis of framing effects. Organizational behavior and human decision processes, Vol. 76, No. 2, pp. 149-188.

Madrian, B. C. and Shea, D. F., 2001. The Power of Suggestion: Inertia in 401 (k) Participation and Savings Behavior. The Quarterly journal of economics, Vol. 116, No. 4, pp. 1149-1187.

McQuinn, A., 2017. The Economics of "Opt-Out" Versus "Opt-In" Privacy Rules. The Information Technology and Innovation Foundation (ITIF).

Momsen, K. and Stoerk, T., 2014. From intention to action: Can nudges help consumers to choose renewable energy? Energy Policy, Vol. 74, No., pp. 376-382.

Nielsen, J. and Pernice, K. 2010. Eyetracking web usability. New Riders, Berkeley, CA.

Ploug, T. et al, 2012. To nudge or not to nudge: cancer screening programmes and the limits of libertarian paternalism. J Epidemiol Community Health, Vol. 66, No. 12, pp. 1193-1196.

Samuelson, W. and Zeckhauser, R., 1988. Status quo bias in decision making. Journal of risk and uncertainty, Vol. 1, No. 1, pp. 7-59.

Simon, H. A., 1957. A Behavioral Model of Rational Choice. Models of Man, Social and Rational: Mathematical Essays on Rational Human Behavior in a Social Setting. New York, Wiley.

Steffen, A. et al, 2019. Upselling by Default: The Effect of Default Options on Travelers' Board and Lodging Choices. Journal of Travel Research, Vol., No., pp. 1-15.

Torres, A. M. et al, 2014. The Identification of Decision Constructs used in Online Transactional Processes. 27th BLED eConference.

Tversky, A. and Kahneman, D., 1981. The framing of decisions and the psychology of choice. Science, Vol. 211, No. 4481, pp. 453-458.

Van Dalen, H. P. and Henkens, K., 2014. Comparing the effects of defaults in organ donation systems. Social science \& medicine, Vol. 106, No., pp. 137-142.

Van Kleef, E. et al, 2018. The effect of a default-based nudge on the choice of whole wheat bread. Appetite, Vol. 121, No., pp. 179-185. 